\title{
Brain Tumor Classification in Magnetic Resonance Images Using Deep Learning and Wavelet Transform
}

\author{
Ahmad M. Sarhan \\ Department of Smart Systems, Middle East University, Amman, Jordan \\ Correspondence to: Ahmad M. Sarhan, ashan@hotmail.com \\ Keywords: Convolutional Neural Network CNN), Wavelet Transform, Image Classification, Brain Cancer, \\ Magnetic Resonance Imaging (MRI) \\ Received: May 10, $2020 \quad$ Accepted: June 14, $2020 \quad$ Published: June 17, 2020 \\ Copyright () 2020 by author(s) and Scientific Research Publishing Inc. \\ This work is licensed under the Creative Commons Attribution International License (CC BY 4.0). \\ http://creativecommons.org/licenses/by/4.0/ \\ (c) (i) Open Access
}

\section{ABSTRACT}

A brain tumor is a mass of abnormal cells in the brain. Brain tumors can be benign (noncancerous) or malignant (cancerous). Conventional diagnosis of a brain tumor by the radiologist is done by examining a set of images produced by magnetic resonance imaging (MRI). Many computer-aided detection (CAD) systems have been developed in order to help the radiologists reach their goal of correctly classifying the MRI image. Convolutional neural networks (CNNs) have been widely used in the classification of medical images. This paper presents a novel CAD technique for the classification of brain tumors in MRI images. The proposed system extracts features from the brain MRI images by utilizing the strong energy compactness property exhibited by the Discrete Wavelet Transform (DWT). The Wavelet features are then applied to a CNN to classify the input MRI image. Experimental results indicate that the proposed approach outperforms other commonly used methods and gives an overall accuracy of $99.3 \%$.

\section{INTRODUCTION}

Brain tumor results from cancer cells that grow uncontrollably in the brain to create a mass of cancer tissue (tumor). The tumor in the brain interferes with brain tasks such as memory, muscle control, and other body operations $[1,2]$. Depending on the type of tumor and its location in the brain, symptoms may include seizures, memory problems, unusual behavior, confusion, vision changes, and balance difficulties [3-5]. Brain cancer may be caused by factors such as exposure to ionizing radiation and a family history of brain cancer [6, 7]. Several studies claim that there is a link between cellphones and brain cancer.

Various treatment options are available for brain cancer patients. The options include radiation therapy, surgery, chemotherapy, or a combination of these treatments [8]. Usually the first stage of treatment given to brain cancer patients is radiotherapy $[9,10]$. The second stage of thereby is a surgery which aims 
to remove all of the tumor. However, if some tumor is left after surgery, which is often the case, then chemotherapy is administered [11, 12].

Magnetic Resonance Imaging (MRI) is the preferred way to diagnose a brain tumor, as it generates more detailed pictures than computerized tomography (CT) scans [13]. MRI is a non-invasive and painless scanning technique. Depending on the type of tumor suspected, the physician may order MRI for the brain, spinal cord, or both [14].

According to the WHO (World Health Organization), brain tumors are classified into two general types: benign (noncancerous) and malignant (cancerous) [15-17]. Malignant tumors are subsequently classified into grades (I through IV.) Grade-I tumor, the lest aggressive type, is called Pilocytic Astrocytoma. Grade-II tumor is a low-grade Astrocytoma. Grade-III tumor is called Anaplastic Astrocytoma. Grade-IV tumor, the most aggressive type, is called Glioblastoma $[18,19]$.

Presented in this paper is a novel brain cancer classification system based on Wavelet decomposition and Convolutional Neural Networks (CNNs). Specifically, this study addresses the classification of three types of brain tumors (meningioma, glioma, and pituitary tumor). T1-weighted contrast-enhanced MRI (CE-MRI) images are adopted in this paper.

Experimental results show that the proposed WCNN system outperforms commonly proposed systems such as the Support Vector Machines (SVM). The proposed system produces a high accuracy rate of $99.3 \%$.

\section{THE STATE OF THE ART IN BRAIN CANCER CLASSIFICATION}

The following detailed classification of brain tumors, according to the WHO, is associated with cell origin and behavior:

- Astrocytoma, which develop from star-shaped cells called astrocytes, are the most common central nervous system (CNS) tumors. Astrocytoma arises anywhere in the spinal cord or brain. In adults, astrocytomas often occur in the cerebrum, the largest part of the brain, that controls speech, movement, learning, thinking, and reading.

- Brain stem gliomas, a high-grade astrocytomas, originate in the brain stem (the lowest part of the brain that connects the spinal cord. to the brain). Brain stem gliomas affect several functions such as breathing, blood pressure, hunger, and body temperature. Tumors in this area can be difficult to treat.

- Glioblastoma, a grade IV astrocytoma, is an aggressive type of CNS tumor that originates in the supportive tissue of the brain and usually affects adults. Glioblastoma, which is considered the most common grade IV brain cancer, may form in any lobe of the brain, but they often develop in the frontal and temporal lobes.

- Meningioma form in the membrane that surrounds the spinal cord and the brain. They are non-cancerous (benign) and are often removed with surgery. Some meningiomas may not need treatment at all.

- Ependymomas often develop in the spinal cord and the lining of the ventricles. They are most common in adolescents and children.

- Oligodendrogliomas, a rare type of slow-growing tumor. They originate in the cells that produce myelin (the fatty coating that keeps nerves in the brain and spinal cord). These tumors often occur in the cerebrum of middle-aged adults. As shown by Table 1, sseveral MRI brain datasets have been used by researchers and are available online.

Conventional cancer detection techniques examine the tissue of the tumor to form a judgment as to the tumor type $[20,21]$. However, many tumors do not possess distinctive morphological characteristics that are essential for differential classification. Hence, the assessment of histopathological and clinical information may lead to a misdiagnosis. Thus, there is a strong demand for automatic methods to perform brain tumor detection and classification.

Computer aided detection (CAD) systems, especially those systems based on machine learning and artificial intelligence (AI) methods, have been widely adopted in cancer detection systems [22]. Some stu- 
dies claim that AI systems have outperformed humans in many imaging areas in medicine $[23,24]$. CAD systems offer many advantages. In addition to providing a fast screening process, CAD systems improve the subjective interpretation of radiologists. As with other fields of medical imaging, CAD systems that are used in brain tumor detection and classification, are significantly based on machine learning and AI software. A variety of CAD techniques have been proposed in the literature for the diagnosis and classification of brain tumor. Segmentation is perhaps the most common preprocessing step among these techniques, and is used to extract the infected region of the brain from the input MRI image.

A review of brain tumor segmentation methods is given by Tiwari [25]. Hakeel et al. used a wireless infrared imaging sensor for brain tumor detection [26]. Maalinii et al. used morphological reconstruction and thresholding for Brain tumor extraction [27]. Jemimma et al. performed brain tumor segmentation using the Watershed algorithm based DAPP features [28]. Yin et al. employed a whale optimization algorithm for brain tumor classification [29]. Gurbină et al. [30] and Shree et al. [31] used Wavelet for brain tumor identification.

\section{MATERIALS AND METHODS}

Proposed in this paper is a Wavelet-based CNN (WCNN) system for brain tumor detection and classification. MRI images, which are the most popular imaging technique for brain tumor scanning, are used in this study. A block diagram showing the main stages of the proposed system is depicted in Figure 1 .

The cascade functions performed by the proposed system start by taking the Wavelet decomposition of the input image I, which is an MRI image and it represents any image in the employed brain tumor dataset.

Magnetic resonance imaging (MRI) is a medical imaging technique that uses a strong magnetic field and radio waves to create detailed images of the body organs and tissues.

MRI allows for the detailed visualization of the brain and spinal cord anatomy in all three planes: axial, sagittal and coronal. The most common MRI relaxation time scans are the T1-weighted (T1) and T2-weighted (T2) scans. If ordered, the MRI can also generate other scans such as T1-weighted contrast-enhanced (T1c), and T2-weighted Fluid Attenuated Inversion Recovery (FLAIR) images [32].

\section{Table 1. MRI brain databases.}

\begin{tabular}{lll}
\hline$\#$ & Database & Location \\
\hline 1 & BRAINIX medical images & https://www.medicalimages.com/search/brain.html \\
2 & TCGA-GBM, TCGA-LGG & $\underline{\text { https://wiki.cancerimagingarchive.net/display/Public/TCGA-LGG }}$ \\
3 & Figshare (Cheng) & $\underline{\text { https://figshare.com/articles/brain tumor dataset/1512427 }}$ \\
4 & Harvard Medical School & $\underline{\text { http://med.harvard.edu/AANLIB/ }}$ \\
5 & Moffitt Cancer Research Center & $\underline{\text { https://moffitt.org/ }}$ \\
6 & BraTS 2013, 2014, 2016, 2018, & $\underline{\text { https://ipp.cbica.upenn.edu/ }}$ \\
2020 &
\end{tabular}

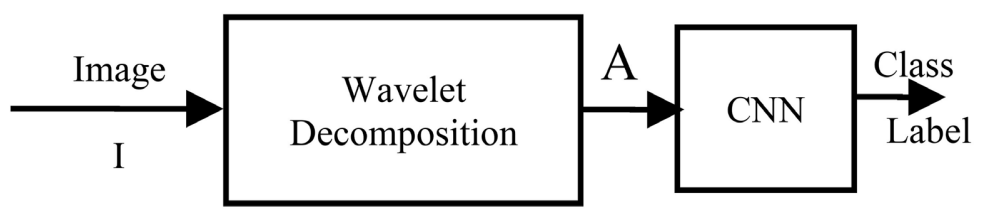

Figure 1. Block diagram of the proposed system. 


\subsection{Image Dataset}

The brain tumor database was obtained from figshare (Cheng) collection. This brain tumor database contains T1-weighted contrast-enhanced images from 233 patients with three types of brain diseases. Specifically, this dataset contains 1426 slices representing glioma, 930 slices representing pituitary tumor, and 708 slices representing meningioma. In this study, only 170 images from each class were used; $70 \%$ for training and $30 \%$ for testing. Table 2 summarizes the dataset details. Sample images from each class of the database are shown in Figure 2.

Unlike most of the brain classification CAD methods which require segmenting the input image before classifying it, the proposed WCNN method operates on the whole input image without doing any segmentation to it. Therefore, the proposed system significantly reduces the time complexity of the classification system and avoids any potential errors which may occur in the segmentation phase. The first phase of the proposed system is to use the Wavelet transform to obtain discriminative features from the input image.

\subsection{Wavelet Transform}

The Wavelet Transform (Wavelet decomposition), is a lossless transform. The transform of an image gives another way of representing the image. It does not change the energy or information content of the image [33]. The Wavelet decomposition tree, shown in Figure 3, illustrates the functions of the Wavelet decomposition transform. The input image, at the first level of decomposition, produces two vectors of coefficients: approximation and detail coefficients. The approximation coefficients represent the low frequency contents of the signal, while the detail coefficients represent the high-frequency components. In the second level of decomposition, the approximation coefficients produce two sets of approximation and detail coefficients, whose lengths are equal to half of the length of the original approximation vector. The process of decomposition further divides the approximation coefficients into two new vectors for each subsequent level of decomposition.

Table 2. Dataset labels and diseases.

\begin{tabular}{cccc}
\hline Disease type & Class label & Number of images & Matrix size \\
\hline meningioma & 1 & 170 & $512 \times 512$ \\
glioma & 2 & 170 & $512 \times 512$ \\
pituitary tumor & 3 & 170 & $512 \times 512$ \\
\hline
\end{tabular}

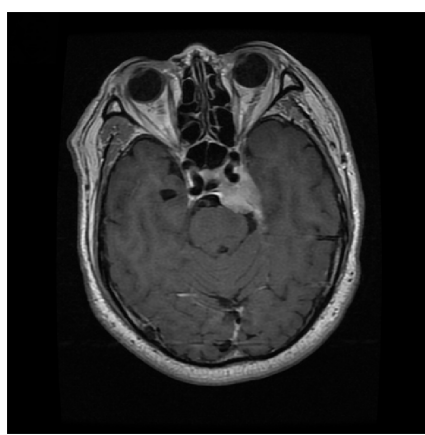

(a)

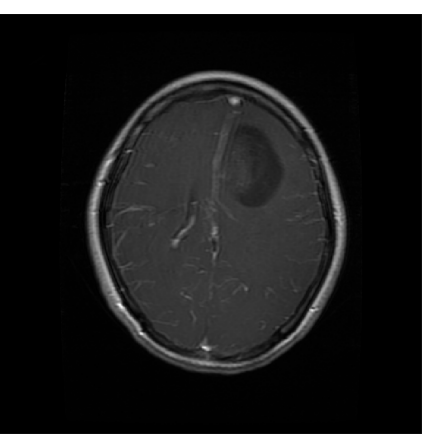

(b)

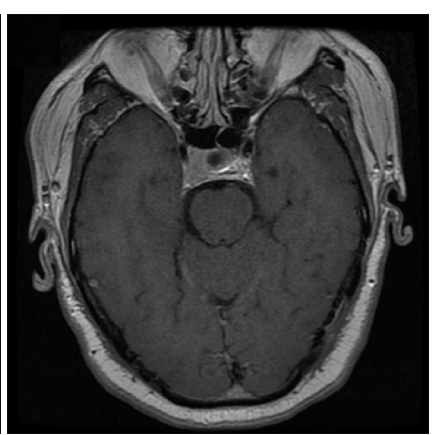

(c)

Figure 2. Sample images from the employed dataset: (a) meningioma, (b) glioma, and (c) pituitary tumor. 


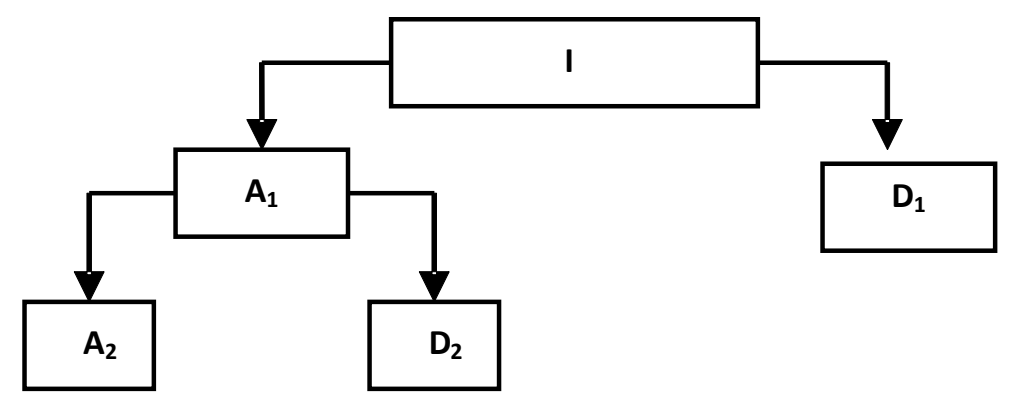

Figure 3. Wavelet decomposition tree. A and D represent the approximation, and detail coefficients.

To show the decomposition operations employed by Wavelet transform, Figure 4 depicts Wavelet decomposition details of a sample image using the Haar Wavelet. The decomposed image was obtained from the meningioma images and is shown in Figure 2(a). The Haar Wavelet which is also known as the $\mathrm{db} 1$ Wavelet, is considered the first and simplest Wavelet. The db1 wavelet looks like a step function [34].

The powerful capability of the Wavelet transforms to compress the image energy makes it suitable for image feature extraction applications [35]. Finally, the feature vector (approximation coefficients) is presented to a CNN and a SVM for classification. The same sets of inputs and outputs are used to train the SVM and the proposed WCNN system.

\subsection{Conventional Neural Network}

A Convolutional Neural Network (CNN) is a special type of Artificial Neural Network (ANN) The original ANNs, such as the multilayer perceptron (MLP), have been very successful in pattern recognition applications [36-38]. ANNs have inspired the creation of CNN, a Deep learning algorithm.

Deep Learning is a branch of Machine Learning that employs Deep Neural Networks; neural networks with many layers. The CNN can be thought of as an ANN where at least one layer applies a convolution operation before it passes its output to the next layer [39]. Commonly, the mean value and the max value functions are used in the convolution operation, but other functions could also be used. CNNs present a quantum leap in the area of image classification and computer vision. A very famous CNN design is the AlexNet [40] which has shown superior performance in general image recognition applications.

The basic structure of a CNN consists of three components: convolutional layer, pooling layer, and output layer. The convolutional layer scans the whole image, using a moving window approach, to create https://www.medicalimages.com/search/brain.html a feature map. The Pooling layer down samples the output of the convolutional layer which reduces the amount of data to be learned. The use of the convolutional and pooling layers is often repeated several times. Fully connected input layer converts the outputs generated by previous layers into a single vector to be applied to the next layer. Fully connected layer produces a weighted sum of the input generated by the feature analysis to predict an output label. The Fully connected layer determines the output class. A typical CNN architecture is depicted in Figure 5.

As indicated by Figure 5, the typical input to a CNN is an image of size $\mathrm{m} \times \mathrm{m} \times \mathrm{r}$, where $\mathrm{r}$ is the number of channels ( $r=1$ for gray-scale and $r=3$ for RGB images). Normally, the CNN has the capability to perform image classification using raw images as direct inputs. However, the implementation of the CNN in the proposed WCNN system uses Wavelet features as inputs to the CNN. This process greatly reduces the number of features; and therefore, makes the learning task of the CNN much easier.

The architecture of the proposed CNN classifier contains five layers: input layer, convolutional layer, max pooling layer, fully connected layer, and output layer. Specifically, the input layer has a size of $1288 \times$ 128 corresponding to the size of the approximation matrices. The output layer had 3 neurons corresponding to the number of classes. Next, we compare the performance of the proposed WCNN system to that of a SVM system. 

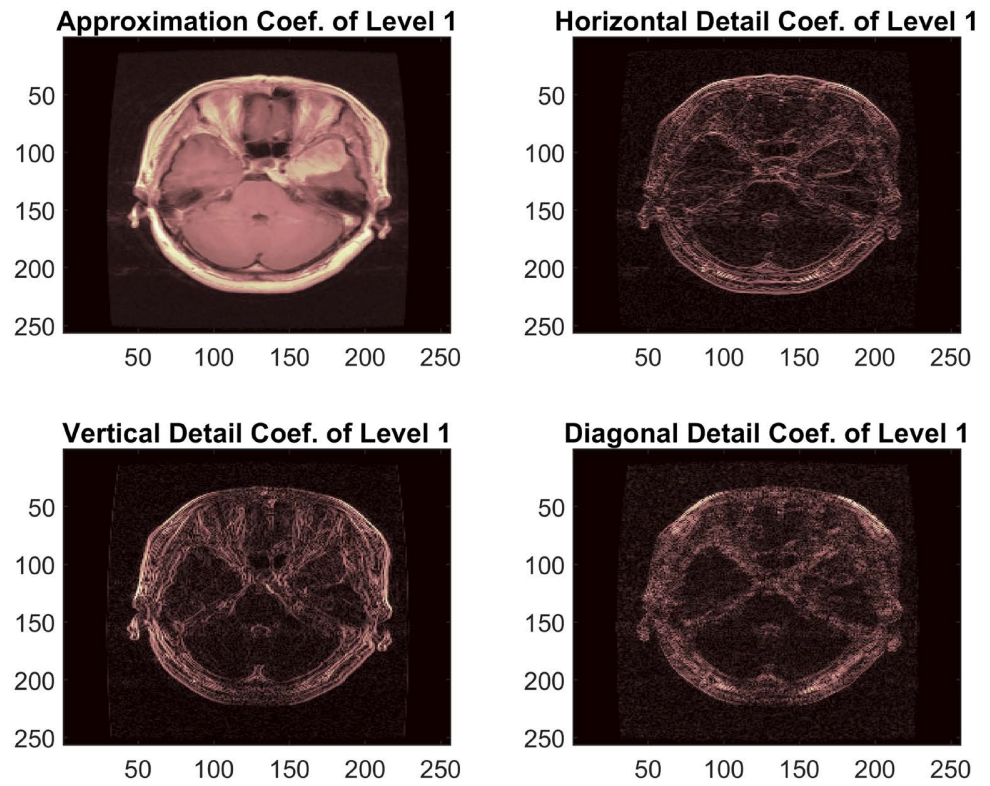

Figure 4. Wavelet decomposition of a meningioma image using the Haar Wavelet.

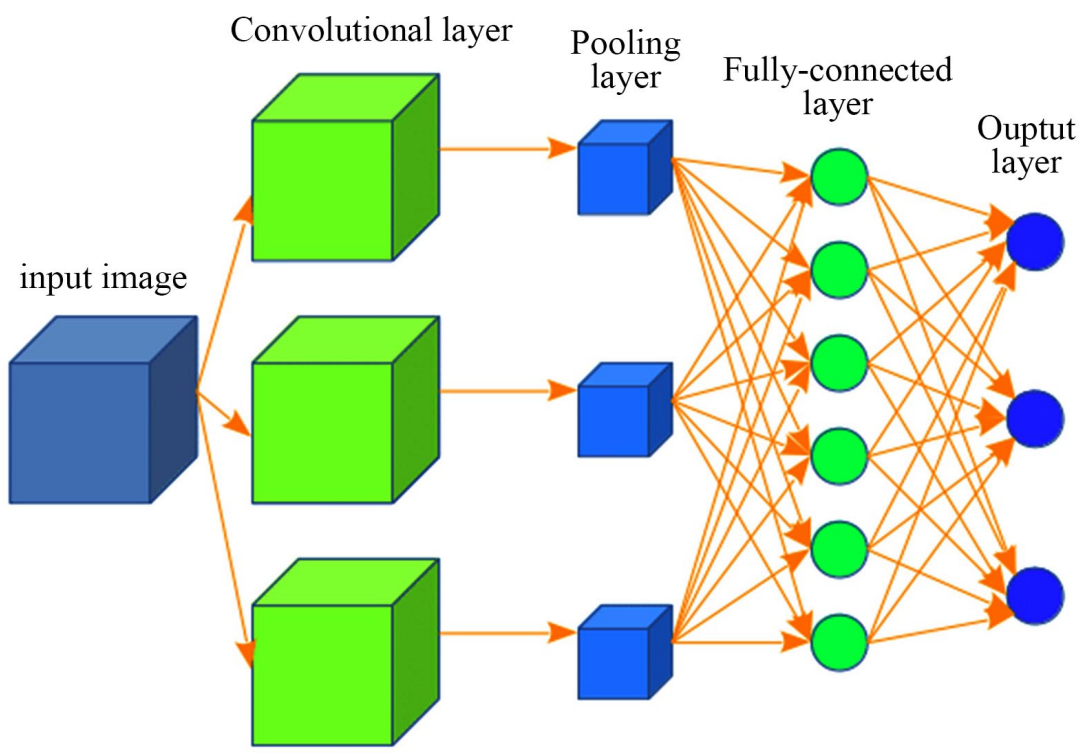

Figure 5. Structure of a typical CNN.

\subsection{Support Vector Machine Implementations}

Like CNNs, SVMs are supervised learning algorithms that have been widely implemented in classification applications. SVMs were originally proposed by Cortes et al. [41, 42]. A SVM was originally designed to be a binary or wo-class classifier. However, SVMs have been altered to tackle data composed of more than two classes [43,44]. SVMs have shown remarkable success in solving linear and non-linear classification problems. As depicted in Figure 6, a SVM classifies data by determining the best hyperplane that isolates the data points of the two classes. In other words, an SVM tries to find the widest possible margin that separates the two classes with no interior data points.

The SVM algorithm implemented here uses the Gaussian kernel defined by: 


$$
k(x, y)=\exp \left(\frac{\|x-y\|^{2}}{2 \sigma^{2}}\right)
$$

where $\sigma$ is a user-defined variance parameter.

\section{DISCUSSION AND RESULTS}

In the experiments, $70 \%$ of the input wavelet matrices (approximation coefficients) were used for training the $\mathrm{CNN}$ and $30 \%$ for testing. The dataset consisted of three classes; with 170 images for each class. The features representing each image is a matrix of Wavelet approximation coefficients. Hence, the dataset consists of 510 matrices. Randomized splitting was used to avoid biasing the results. The traces of accuracy of the proposed system are shown in Figure 7.

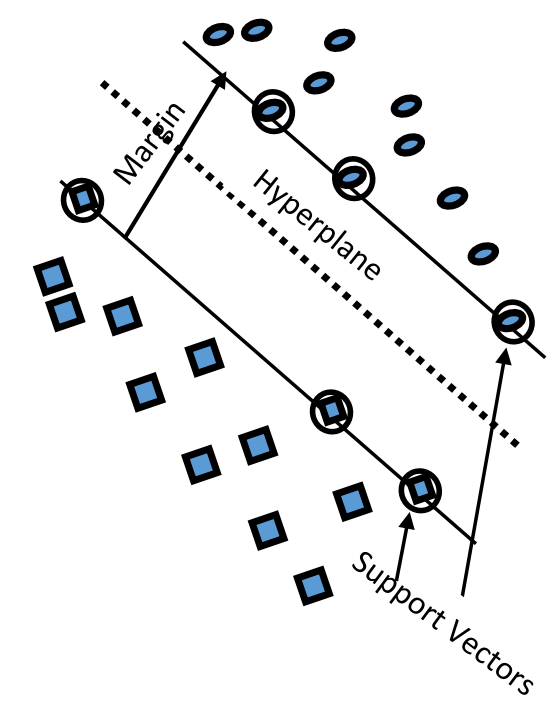

Figure 6. Support vector machine.

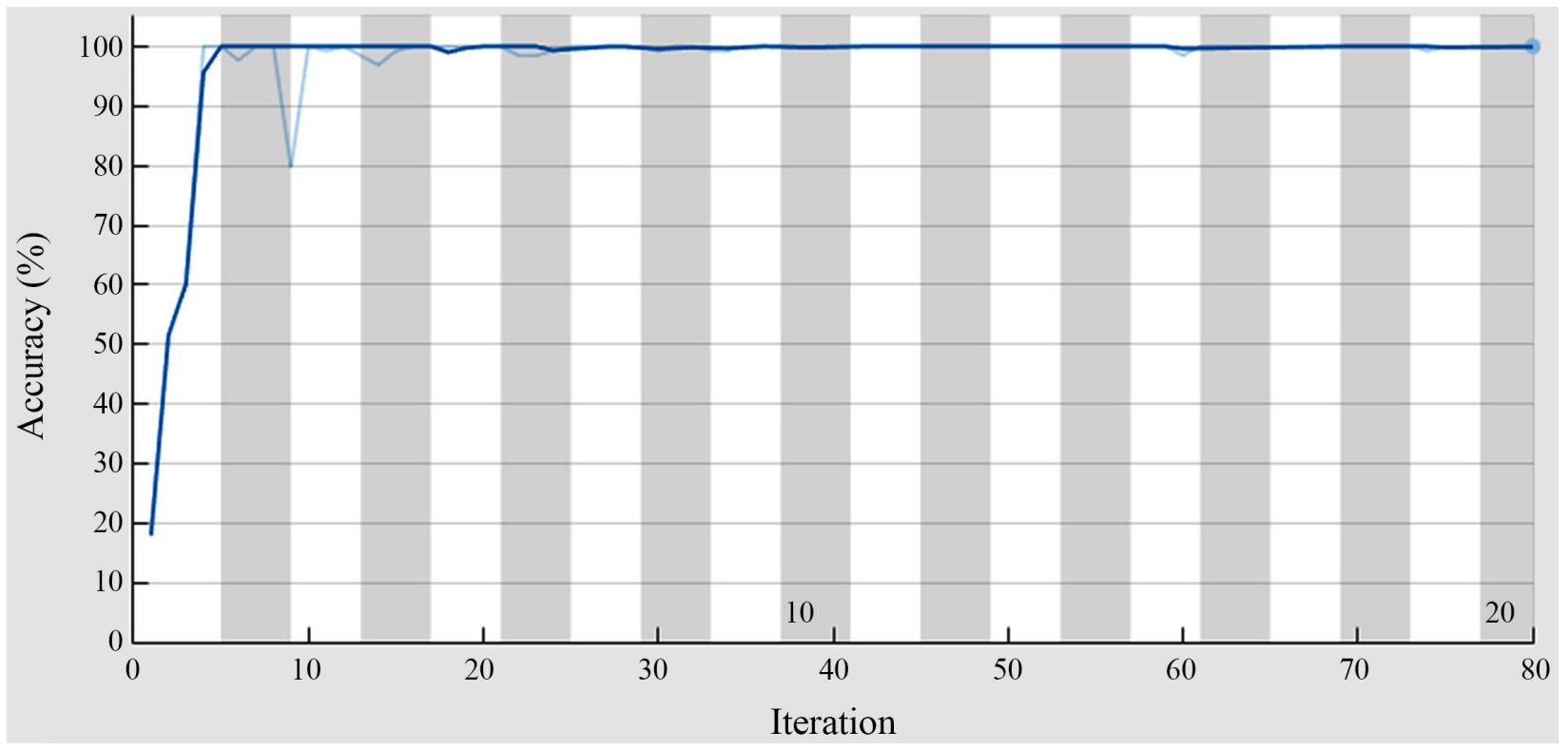

Figure 7. Traces of accuracy during training. 
The maximum success rate (accuracy) of the proposed WCNN system for this experiment is $99.3 \%$, indicating that the approximation coefficients carry highly distinctive information about the Brain image. To show the validity of the proposed system, its accuracy is compared to the SVM classifier. When operating on the same feature matrices as the proposed WCNN system, the SVM system produced an accuracy of $98.5 \%$.

Several statistical measures are used to analyze the performance of the proposed WCNN system. Specifically, the performance of the proposed algorithm is evaluated by computing the percentages of sensitivity (SE), specificity (SP) and accuracy (ACC) as follows:

Sensitivity: is the fraction of real events that are correctly detected among all real events and is given by:

$$
\mathrm{SE}=\frac{\mathrm{TP} \times 100}{(\mathrm{TP}+\mathrm{FN})}
$$

Specificity is defined as the fraction of nonevents that are correctly rejected and is given by:

$$
\mathrm{SP}=\frac{\mathrm{TN} \times 100}{(\mathrm{TN}+\mathrm{FP})}
$$

Accuracy is the fraction of the real events that are correctly detected and the non-events that are correctly rejected, among all events and non-events and is defined as:

$$
\mathrm{ACC}=\frac{(\mathrm{TP}+\mathrm{TN}) \times 100}{(\mathrm{TP}+\mathrm{TN}+\mathrm{FP}+\mathrm{FN})}
$$

where,

FP: number of false positive specimens (predicts non-tumor as tumor).

TP: number of true positive specimens (predicts tumor as tumor).

FN: number of false negative specimens (predicts tumor as non-tumor).

TN: number of true negative specimens (predicts non-tumor as non-tumor).

The prevalence is determined from the sensitivity, specificity, and accuracy using the following equation:

$$
\text { Accuracy }=(\text { sensitivity })(\text { prevalence })+(\text { specificity })(1-\text { prevalence })
$$

The calculated SE, SP, AC, and prevalence are given in Table 3, which shows that the proposed system produces high sensitivity and specificity values, indicating that the system is robust and reliable.

\section{CONCLUSION}

In this paper, a novel approach to the classification of Brain cancer using Deep Neural Network is presented and developed. Most of the systems that are currently proposed in the literature, segment the input Brain image before applying it to the $\mathrm{CNN}$ classifier. The proposed CNN system processes the whole Brain image with Wavelet decomposition, without doing any segmentation to the input image. Hence, the proposed system has a lower time complexity than the other systems proposed in the literature. Wavelet decomposition highly reduces the dimensions of the input image, which in turn, simplifies the work of the CNN classifier. The proposed system classifies the input Brain image to one of three classes: meningioma, glioma, and pituitary tumor.

Table 3. Performance metrics of the proposed system.

\begin{tabular}{ccccc}
\hline No. of cases & SE & SP & ACC & Prevalence \\
\hline 100 & $97.4 \%$ & $95.5 \%$ & $99.3 \%$ & 2 \\
\hline
\end{tabular}


The Brain images are MRI scans taken from the Figshare (Cheng) database. To show the validity and robustness of the proposed system, its performance is compared to the SVM classifier. Both the proposed WCNN system and the SVM classifier received the same feature vectors as input.

Experimental tests on the Figshare (Cheng) database achieved $99.3 \%$ of recognition accuracy using a decomposition level of two and the Haar wavelet. Simulation results have indicated that the proposed system always produces higher success rates than the SVM system.

\section{CONFLICTS OF INTEREST}

The author declares no conflicts of interest regarding the publication of this paper.

\section{REFERENCES}

1. The American Cancer Society. https://www.cancer.org

2. Komori, T. (2020) Updating the Grading Criteria for Adult Diffuse Gliomas: Beyond the WHO2016CNS Classification. Brain Tumor Pathology, 37, 1-4. https://doi.org/10.1007/s10014-020-00358-y

3. Koriyama, S., Nitta, M., Kobayashi, T., et al. (2018) A Surgical Strategy for Lower Grade Gliomas Using Intraoperative Molecular Diagnosis. Brain Tumor Pathology, 35, 159-167. https://doi.org/10.1007/s10014-018-0324-1

4. Ogawa, K., Kurose, A., Kamataki, A., et al. (2020) Giant Cell Glioblastoma Is a Distinctive Subtype of Glioma Characterized by Vulnerability to DNA Damage. Brain Tumor Pathology, 37, 5-13.

https://doi.org/10.1007/s10014-019-00355-w

5. Asano, K., Kurose, A., Kamataki, A., et al. (2018) Importance and Accuracy of Intraoperative Frozen Section Diagnosis of the Resection Margin for Effective Carmustine Wafer Implantation. Brain Tumor Pathology, 35, 131-140. https://doi.org/10.1007/s10014-018-0320-5

6. Park, S.-H., et al. (2017) Molecular Testing of Brain Tumor. Journal of Pathology and Translational Medicine, 51, 205-223. https://doi.org/10.4132/jptm.2017.03.08

7. Góes, P., Santos, B.F.O., Suzuki, F.S., et al. (2017) Necrosis Is a Consistent Factor to Recurrence of Meningiomas: Should It Be a Stand-Alone Grading Criterion for Grade II Meningioma? Journal of Neuro-Oncology, 137, 331-336. https://doi.org/10.1007/s11060-017-2721-4

8. Sasaki, S., Tomomasa, R., Nobusawa, S., et al. (2019) Anaplastic Pleomorphic Xanthoastrocytoma Associated with an H3G34 Mutation: A Case Report with Review of Literature. Brain Tumor Pathology, 36, 169-173. https://doi.org/10.1007/s10014-019-00349-8

9. Tan, C.L., Vellayappan, B., Wu, B., et al. (2018) Molecular Profiling of Different Glioma Specimens from an Ollier Disease Patient Suggests a Multifocal Disease Process in the Setting of IDH Mosaicism. Brain Tumor Pathology, 35, 202-208. https://doi.org/10.1007/s10014-018-0327-y

10. Yamasaki, T., Sakai, N., Shinmura, K., et al. (2018) Anaplastic Changes of Diffuse Leptomeningeal Glioneuronal Tumor with Polar Spongioblastoma Pattern. Brain Tumor Pathology, 35, 209-216.

https://doi.org/10.1007/s10014-018-0326-Z

11. Girolami, I., Cima, L., Ghimenton, C., et al. (2018) NRASQ61K Mutated Diffuse Leptomeningeal Melanomatosis in an Adult Patient with a Brief Review of the So-Called "Forme Fruste" of Neurocutaneous Melanosis. Brain Tumor Pathology, 35, 217-223. https://doi.org/10.1007/s10014-018-0328-x

12. Louis, D.N. (2018) A Feast of Reviews about Brain and Pituitary Tumor Pathology. Brain Tumor Pathology, 35, 49-50. https://doi.org/10.1007/s10014-018-0315-2

13. Jerban, S., Chang, E.Y. and Du, J. (2020) Magnetic Resonance Imaging (MRI) Studies of Knee Joint under Mechanical Loading: Review. Magnetic Resonance Imaging, 65, 27-36. https://doi.org/10.1016/j.mri.2019.09.007

14. Wadhwa, A., Bhardwaj, A. and Singh Verma, V. (2019) A Review on Brain Tumor Segmentation of MRI Im- 
ages. Magnetic Resonance Imaging, 61, 247-259. https://doi.org/10.1016/j.mri.2019.05.043

15. Nishioka, H. and Inoshita, N. (2018) New WHO Classification of Pituitary Adenomas (4th Edition): Assessment of Pituitary Transcription Factors and the Prognostic Histological Factors. Brain Tumor Pathology, 35, 57-61. https://doi.org/10.1007/s10014-017-0307-7

16. Iuchi, T., Sugiyama, T., Ohira, M., et al. (2018) Clinical Significance of the 2016 WHO Classification in Japanese Patients with Gliomas. Brain Tumor Pathology, 35, 71-80. https://doi.org/10.1007/s10014-018-0309-0

17. Shibuya, M. (2018) Welcoming the New WHO Classification of Pituitary Tumors 2017: Revolution in TTF-1-Positive Posterior Pituitary Tumors. Brain Tumor Pathology, 35, 62-70.

https://doi.org/10.1007/s10014-018-0311-6

18. Akagi, Y., Yoshimoto, K., Hata, N., et al. (2018) Reclassification of 400 Consecutive Glioma Cases Based on the Revised 2016 WHO Classification. Brain Tumor Pathology, 35, 81-89.

https://doi.org/10.1007/s10014-018-0313-4

19. Kuwahara, K., Ohba, S., Nakae, S., et al. (2019) Clinical, Histopathological, and Molecular Analyses of IDH-Wild-Type WHO Grade II-III Gliomas to Establish Genetic Predictors of Poor Prognosis. Brain Tumor Pathology, 36, 135-143. https://doi.org/10.1007/s10014-019-00348-9

20. Barresi, V., Lionti, S., Caliri, S., et al. (2018) Histopathological Features to Define Atypical Meningioma: What Does Really Matter for Prognosis? Brain Tumor Pathology, 35, 168-180.

https://doi.org/10.1007/s10014-018-0318-z

21. Nambirajan, A., Malgulwar, P.B., Sharma, A., et al. (2019) Clinicopathological Evaluation of PD-L1 Expression and Cytotoxic T-Lymphocyte Infiltrates across Intracranial Molecular Subgroups of Ependymomas: Are These Tumors Potential Candidates for Immune Check-Point Blockade? Brain Tumor Pathology, 36, 152-161.

https://www.springermedizin.de https://doi.org/10.1007/s10014-019-00350-1

22. Challen, R., Denny, J., Pitt, M., Gompels, L., Edwards, T. and Tsaneva-Atanasova, K. (2019) Artificial Intelligence, Bias and Clinical Safety. BMJ Quality \& Safety, 28, 231-237.

https://qualitysafety.bmj.com/content/28/3/231

https://doi.org/10.1136/bmjqs-2018-008370

23. Crawford, K. and Calo, R. (2016) There Is a Blind Spot in AI Research. Nature, 538, 311-313. https://doi.org/10.1038/538311a

24. Lallas, A. and Argenziano, G. (2018) Artificial Intelligence and Melanoma Diagnosis: Ignoring Human Nature May Lead to False Predictions. Dermatology Practical \& Conceptual, 8, 249-251.

https://dpcj.org/index.php/dpc/article/view/dermatol-pract-concept-articleid-dp0804a01

https://doi.org/10.5826/dpc.0804a01

25. Tiwari, A., Srivastava, S. and Pant, M. (2019) Brain Tumor Segmentation and Classification from Magnetic Resonance Images: Review of Selected Methods from 2014 to 2019. Pattern Recognition Letters, 131, 244-260. https://doi.org/10.1016/j.patrec.2019.11.020

26. hakeel, P.M., Tobely, T.E.E.L., Al-feel, H., Manogaran, G. and Baskar, S. (2019) Neural Network Based Brain Tumor Detection Using Wireless Infrared Imaging Sensor. IEEE Access, 7, 5577-5588.

https://ieeexplore.ieee.org/document/8599180

https://doi.org/10.1109/ACCESS.2018.2883957

27. Maalinii, G.B. and Jatti, A. (2018) Brain Tumour Extraction Using Morphological Reconstruction and Thresholding. Materials Today Proceedings, 5, 10689-10696. https://doi.org/10.1016/j.matpr.2017.12.350

28. Jemimma, T.A. and Vetharaj, Y.J. (2018) Watershed Algorithm Based DAPP Features for Brain Tumor Segmentation and Classification. 2018 International Conference on Smart Systems and Inventive Technology, Ti- 
runelveli, 13-14 December 2018, 155-158. https://ieexplore.ieee.org/document/8748436 https://doi.org/10.1109/ICSSIT.2018.8748436

29. Yin, B., Wang, C. and Abza, F. (2019) New Brain Tumor Classification Method Based on an Improved Version of Whale Optimization Algorithm. Biomedical Signal Processing and Control, 56, Article ID: 101728. https://doi.org/10.1016/j.bspc.2019.101728

30. Gurbină, M., Lascu, M. and Lascu, D. (2019) Tumor Detection and Classification of MRI Brain Image Using Different Wavelet Transforms and Support Vector Machines. 42nd International Conference on Telecommunications and Signal Processing, Budapest, 1-3 July 2019, 505-508.

https://ieeexplore.ieee.org/abstract/document/8769040

https://doi.org/10.1109/TSP.2019.8769040

31. Shree, N.V. (2018) Identification and Classification of Brain Tumor MRI Images with Feature Extraction Using DWT and Probabilistic Neural Network. Brian Informatics, 5, 23-30. https://doi.org/10.1007/s40708-017-0075-5

32. Stachera, M. and Sznajder, K. (2014) Magnetic Resonance in Human Brain Examinations: A Brief Outline of the Techniques. Cornell University, Utrecht. https://www.researchgate.net/publication

33. Sarhan, A. and Al-Dosari, R. (2017) Mammogram Classification Using Discrete Wavelet Transform Features and a Novel Vector Quantization Technique for Breast Cancer Detection. British Journal of Applied Science \& Technology, 19, 20-35. https://doi.org/10.9734/BJAST/2017/30420

34. Sarhan, A. (2014) A WPD Scanning Technique for Iris Recognition. International Journal of Computer Applications, 85, 6-12. https://doi.org/10.5120/14907-3446

35. Sarhan, A. (2013) Wavelet-Based Feature Extraction for DNA Microarray Classification. Artificial Intelligence Review, 39, 237-249. https://doi.org/10.1007/s10462-011-9269-z

36. Buragga, K., Aljahdali, S. and Sarhan, A. (2015) An Efficient Technique for Iris Recognition Using Wavelets and Artificial Neural Networks. Proceedings of CATA 2015, Hawaii, 9-11 March 2015.

https://pdfs.semanticscholar.org/bfaf/868446310c78bd0497a5ca60f4ae23ad685d.pdf

37. Pal, S. and Mitra, S. (1992) Multilayer Perceptron, Fuzzy Sets, and Classification. IEEE Transactions on Neural Networks, 3, 683-697. https://doi.org/10.1109/72.159058

38. Sarhan, A. and Al-Helalat, O. (2007) Arabic Character Recognition Using Artificial Neural Networks and Statistical Analysis. Proceedings of the ICCESSE Conference, May 2007, 32-36. https://pdfs.semanticscholar.org/b92d/2f528f95bd72f489fbfcf82167f7da100394.pdf

39. Sarhan, A. (2020) Lung Cancer Classification in Computed Tomography Images Using Wavelet and Convolutional Neural Network. Journal of Biomedical Science and Engineering, 13, 33-48.

40. Han, X., Zhong, Y., Cao, L. and Zhang, L. (2017) Pre-Trained AlexNet Architecture with Pyramid Pooling and Supervision for High Spatial Resolution Remote Sensing Image Scene Classification. Remote Sensing, 9, 848. https://doi.org/10.3390/rs9080848

41. Cortes, C. and Vapnik, V. (1995) Support-Vector Networks. Machine Learning, 20, 273-297. https://doi.org/10.1007/BF00994018

42. Segera, D., Mbuthia, M. and Nyete, A. (2019) TI Particle Swarm Optimized Hybrid Kernel-Based Multiclass Support Vector Machine for Microarray Cancer Data Analysis. BioMed Research International, 2019, Article ID: 4085725 . https://doi.org/10.1155/2019/4085725

43. Sarhan, A.M. (2010) A Novel Gene-Based Cancer Diagnosis with Wavelets and Support Vector Machines. European Journal of Scientific Research, 46, 488-502.

44. Wang, L. (2005) Support Vector Machines: Theory and Applications Vector Machines. Computer Science Vol. 177, Springer, Berlin, 6221. https://www.springer.com/gp/book/9783540243885 